\title{
Probiotic beverage: the potential of anti-diabetes within kombucha tea made from sea grapes (Ceulerpa racemosa) containing high antioxidant and polyphenol total
}

\author{
P.S. Augusta ${ }^{1}$, F. Nurkolis ${ }^{2}$, S.L. Noor ${ }^{3}$, H.K. Permatasari ${ }^{1}$, Hardinsyah ${ }^{4}$, N.A. Taslim ${ }^{5}$, \\ S.C. Batubara ${ }^{6}$, N. Mayulu ${ }^{7}$, D.S. Wewengkang ${ }^{8}$ and H. Rotinsulu ${ }^{8}$ \\ ${ }^{1}$ Medicine, Faculty of Medicine, Brawijaya University, Malang, Indonesia, \\ ${ }^{2}$ Department of Biological Sciences, Faculty of Sciences and Technology, State Islamic University of Sunan Kalijaga, \\ Yogyakarta, Indonesia, \\ ${ }^{3}$ University College London, UK, \\ ${ }^{4} I P B$ University, Bogor, Indonesia, \\ ${ }^{5}$ Faculty of Medicine, Hasanuddin University, Makassar, Indonesia, \\ ${ }^{6}$ Faculty of Food Technology and Health, Sahid University, Jakarta, Indonesia, \\ ${ }^{7}$ Food and Nutrition Department of Sam Ratulangi University, Kampus Unsrat Bahu Street, Manado, Indonesia and \\ ${ }^{8}$ Pharmacy Department of Sam Ratulangi University, Kampus Unsrat Bahu Street, Manado, Indonesia
}

Seagrapes (Ceulerpa racemosa) are algae spread widely in Indonesian waters, yet the use of seagrapes is rare - economic or health products $^{(1)}$. Commonly, it is just consumed as fresh vegetables or salads. Several studies show sea grapes is the source of macro-and micronutrient as well as high-quality protein, soluble dietary fibre, vitamins, minerals, phytochemicals, and non-saturated fatty acids. Those nutrients can prevent the body from chronic disease and aging. Therefore, this study aims to process seagrapes to fermentation tea or kombucha tea and determine highest levels of antioxidants and total polyphenols.

Seagrapes can be obtained in North Sulawesi waters at a depth around 10-20 meters above sea level. Fresh seagrapes are drained at room temperature for about 5 hours, in terms of reducing the water level. Next, the drained seagrapes are crushed using a blender. The basic formulation for all samples of Kombucha tea is 25 grams of seagrapes, $50 \mathrm{~mL}$ of water, and 10 grams of SCOBY gel (Symbiotic Culture of Bacteria and Yeast), with a diameter of $16 \mathrm{~cm}$. There are 6 samples with different treatments or additions $(\mathrm{S} 1=100$ grams cane sugar, S2 $=100$ grams Trigona sapiens honey, S3 = 100 grams brown sugar, S4 = 100 grams Stevia powder, S5 = 100 grams rock sugar, and S6 = control / without any addition). Each sample treatments also has variations in the concentration of v/v SCOBY starter solution, which are V1 10\%, V2 15\%, and V3 20\%. All samples are put in 1000-mL bottles and anaerobic condition with room temperature (20-25 C) for 12 days. The determination of water content used was the AOAC drying method (Thermogravimetry) and also ash content was carried out using the AOAC. Antioxidant activities can be determined using 2.2-diphenyl-1-picrylhydrazyl (DPPH) and polyphenol total can use using visible light spectrophotometry method Folin-Ciocalteu reagent. The testing of each sample treatments with variations in the concentration of v/v SCOBY starter solution ((S1V1, S1V2, S1V3); (S2V1, S2V2, S2V3); (S3V1, S3V2, S3V3), (S4V1, S4V2, S4V3); (S5V1, S5V2, S5V3); (S6V1, S6V2, S6V3)) is done in triplicates (triple). The formula with the highest of antioxidant activity and polyphenol total is then tested for the alcohol and $\mathrm{pH}$ levels. This study is undertaken in the Pharmacy Laboratory, Sam Ratulangi University.

In S1, V2 is the high in antioxidant activity $42.04 \pm 0.25 \%$ and the total polyphenol high level in V3 with the number $25.78 \pm 0.22 \%$. In S2, the highest level of antioxidant activity is V3 $(49.33 \pm 0.45 \%)$ and polyphenol total is high in V3 $(29.80 \pm 0.32 \%)$. In S3, the highest level of antioxidant activity in V3 (40.28 $\pm 1.46 \%)$ and polyphenol total is high in V3 $(22.95 \pm 0.07 \%)$. In S4, the highest level of antioxidant activity in V1 $(40.03 \pm 0.79 \%)$ and polyphenol total is high in V2 (21.27 $\pm 0.54 \%)$. In S5, the highest level of antioxidant activity in V3 $(41.33 \pm 0.30 \%)$ and polyphenol total is high in V3 $(23.38 \pm 0.35 \%)$. In S6 or control group, the highest level of antioxidant activity in V3 $(35.66 \pm 0.55 \%)$ and polyphenol total is high in V3 $(19.58 \pm 0.13 \%$.) The sample with the best pairs (high both antioxidant activity and polyphenol total) is S2 (sample with treatment 2 or with addition of 100 grams Trigona sapiens honey) and with $20 \%$ variation of SCOBY starter solution or V3 (S2V3). S2V3 has ash and water level of $7.07 \pm 0.15 \%$ and $44.85 \pm 0.96 \%$ respectively, as well as alcohol and pH levels in $0.62 \pm 0.50 \%$ and $4.78 \%$ respectively. The treatments of adding different sweeteners and different variations of the SCOBY starter solution, also resulted in different antioxidant activity and polyphenols total in each sample.

Seagrapes are possible to be processed as a probiotic beverage, high antioxidant, and polyphenol, also potential anti-diabetic (by looking at its antioxidant and polyphenol in terms of improving lipid profiles and blood sugar fluctuation ${ }^{(1,2)}$ ). Formulation of Kombucha tea is optimum in S2V3 (highest antioxidant activity and polyphenol total) with the safe concentration of alcohol and pH as the requirements to be judged as probiotic. This formula can be a reference for manufacturers to produce Kombucha tea from seagrapes.

\section{Acknowledgements}

All authors contributed to the writing and revisions contained in the abstract as Original Communications. We thank all of the contributors for their outstanding help in formatting the abstract, especially Fahrul Nurkolis who has fully assisted in laboratory tests.

\section{References}

1. Qudus B, Aroyehun A, Abdul Razak S, Palaniveloo K, et al. (2020) Foods 9(9), 1313

2. Neuenschwander M, Ballon A, Weber K S, et al. (2019) BMJ 366, 12368 